\title{
A unique foot-worn device for patients with degenerative meniscal tear
}

\author{
Avi Elbaz $\cdot$ Yiftah Beer $\cdot$ Ehud Rath $\cdot$ Guy Morag • \\ Ganit Segal · Eytan M. Debbi · Daniel Wasser • \\ Amit Mor $\cdot$ Ronen Debi
}

Received: 28 November 2011/Accepted: 17 April 2012/Published online: 4 May 2012

(C) The Author(s) 2012. This article is published with open access at Springerlink.com

\begin{abstract}
Purpose The purpose of the current study was to assess the effects of a new foot-worn device on the gait, physical function and pain in patients suffering from knee osteoarthritis (OA) who had a low-impact injury to the medial meniscus causing a degenerative meniscal tear.

Methods A retrospective analysis of 34 patients with knee $\mathrm{OA}$ and a degenerative medial meniscal tear was performed. Patients underwent a gait evaluation, using an electronic walkway mat, and completed the SF-36 health survey and the WOMAC questionnaire at baseline and after 3 and 12 months of therapy. AposTherapy is a functional, biomechanical, non-invasive rehabilitation therapy consisting of a foot-worn device that is individually calibrated to each patient and is used during activities of daily living. Repeated-measures analyses were performed to compare gait parameters and self-evaluation questionnaires between baseline, and 3 and 12 months.

Results Significant improvements were found in gait velocity, step length and single-limb support of the involved knee following 12 weeks of therapy (all $p<0.01$ ), alongside
\end{abstract}

\footnotetext{
A. Elbaz · G. Segal · E. M. Debbi - D. Wasser · A. Mor

AposTherapy Research Group, Herzliya, Israel

Y. Beer

Department of Orthopedic Surgery,

Assaf HaRofeh Medical Center, Zerifin, Israel

E. Rath · G. Morag

Department of Orthopedic Surgery, Sourasky Medical Center,

Tel-Aviv, Israel

R. Debi $(\bowtie)$

Department of Orthopedic Surgery, Barzilay Medical Center,

Hahistadrout St 2, 78278 Ashkelon, Israel

e-mail: researchdept10@gmail.com
}

an improvement in limb symmetry. These results were maintained at the 12-month follow-up examination. Significant improvements were also found in all three domains of the WOMAC index (pain, stiffness and physical function) and in the SF-36 Physical Health Scale and the SF-36 Mental Health Scale (all $p<0.01$ ).

Conclusions Patients with knee OA and a degenerative medial meniscal tear using a biomechanical foot-worn device for a year showed improvement in gait, physical function and pain. Based on the findings of this study, it can be postulated that this biomechanical device might have a positive effect on this population.

Level of evidence Therapeutic study, Level IV.

Keywords Gait - Meniscal tear - Physical function . Pain · Osteoarthritis

\section{Introduction}

Meniscal tears are the leading cause of knee injury [34]. In the United States, $60 \%$ of people over the age of 65 diagnosed with knee osteoarthritis (OA) suffer from chronic meniscal damage [13]. Meniscal tears have serious consequences as patients suffer from significant pain and a profound decline in their quality of life and physical function [34].

A variety of therapies exist to treat meniscal tears, ranging from pharmaceutical treatment [38] to physical therapy $[15,24]$ to surgery $[2,22,30]$. The most common invasive therapy has traditionally been meniscectomy [16], though the procedure has been reported to not halt the progression of cartilage destruction and premature OA [6, 29,31], and it has even been suggested that the procedure may accelerate the development of OA [34-36]. Alongside this, Englund et al. 
[14] found that in knees without surgery, meniscal damage is a potent risk factor for the development of radiographic OA. In addition, recent work has found meniscectomy not to be superior to conservative treatment in regard to pain sensation, function and quality of life [24].

Gait analysis has been shown to be an objective measurement tool to assess pain, function and quality of life [9]. A common shortcoming of both surgical and nonsurgical therapies (e.g. pharmaceutical management and physiotherapy) has been that proper limb symmetry and support during gait is rarely re-established [7, 37]. Earlier works have found that patients with abnormal gait patterns often suffer from impaired physical function [23] and pain [29]. Step length and single-limb support (SLS) are gait parameters that can demonstrate limb symmetry.

AposTherapy is a treatment that has been shown to improve gait patterns, physical function and pain in patients with orthopaedic pathologies, such as knee OA [3, 11, 20] and nonspecific low back pain [10]. These earlier works suggest that the changes in gait patterns and clinical findings seen with AposTherapy are due to small alterations in the centre of pressure that changes the vector trajectory and leads to reduced pain [18, 19, 21]. Based on AposTherapy principles, proper biomechanical alignment leading to reduced pain and neuromuscular training under controlled perturbation, it may be assumed that patients suffering from meniscal tears may benefit from this treatment and might avoid surgery.

The aim of the current study was to describe the effect of AposTherapy on gait patterns of patients with knee OA who had a low-impact injury to the meniscus causing a degenerative meniscal tear, alongside an analysis of the physical function, pain and quality of life condition throughout the therapy.

\section{Materials and methods}

The study population composed of 34 patients (18 women). All patients were diagnosed with medial compartment knee OA by their physician and had a low-energy indirect injury to the knee, causing pain and functional limitation. Patients were diagnosed with a large complex medial meniscal tear related to the injury accompanied with bone bruise of the knee via magnetic resonance imaging (MRI) [5, 28]. Symptomatically, patients reported a sudden increase in their knee pain and limitation in function following the injury. The average age was 56.1 years $( \pm 11.1$ years) with an average body mass index of $28.5\left( \pm 4.1 \mathrm{~kg} / \mathrm{m}^{2}\right)$. The patient's functional severity was characterized using the functional classification scheme of Elbaz et al.: $6 \%$ fell into Q1, $3 \%$ fell into Q2, $26 \%$ fell into Q3, $39 \%$ fell into Q4 and $26 \%$ fell into Q5. According to this classification, patients who fall into Q1 are characterized with poor walking abilities and high levels of pain and functional limitation, and patients who fall into Q5 are characterized with normal walking abilities and low levels of pain and functional limitation [12]. The patients sought medical care at AposTherapy Center in Herzliya, Israel. A retrospective search on the centre's database for eligible patients was performed. Eligibility was defined according to the following criteria: diagnosed with knee OA according to American College of Rheumatology (ACR) clinical criteria [1], having a low-energy indirect injury to the medial meniscus of the knee diagnosed by MRI within the 3 months prior to arriving at the clinic, and completing a gait test and questionnaires at baseline and after 3 months and after 12 months. Exclusion criteria were (1) acute septic arthritis; (2) inflammatory arthritis; (3) corticosteroid injection within 3 months of the study; (4) avascular necrosis of the knee; (5) joint replacement; (6) neuropathic arthropathy; (7) history of pathological osteoporotic fracture; (8) symptomatic degenerative arthritis in lower limb joints other than the knees. The head researcher used these criteria to determine the inclusion or exclusion of patients from the existing database. The study was approved by the Helsinki committee of Assaf Harofeh Medical Center, Zerifin, Israel (ID no. 141/08). The study was registered in the NIH clinical trial registration system (No. NCT00767780).

\section{Gait analysis}

The GaitMat $^{\mathrm{TM}}$ system (E.Q., Inc. Chalfont, PA, USA) was used to measure gait spatiotemporal parameters. The computerized mat is an electronic walkway carpet that is $3.84 \mathrm{~m}$ long. The spatiotemporal characteristics are measured, processed and stored on a PC running the GaitMat software (version 2). The GaitMat ${ }^{\mathrm{TM}}$ system is a reliable tool to measure gait variables with significant accuracy, validly and reliably [4]. Temporal measurements taken simultaneously by the Gait Mat and Vicon had an ICC value of 0.99 , indicating excellent reliability. Distance measurements taken by the two systems had an ICC value of 0.24 , indicating poor to moderate reliability. The mean difference between distance measures was $11.7 \mathrm{~mm}$, a difference that would be clinically significant only for support base measurements. The measurement accuracy is to the one decimal point [4].

Physical function and pain assessment

The Western Ontario and McMaster Universities Osteoarthritis Index (WOMAC) was used to assess pain and physical function. It consists of a series of 24 self-administered questions measuring the pain, joint stiffness and 
physical function of a person with knee OA and is scored from 0 to $100 \mathrm{~mm}$ on a visual analogue scale (VAS), where 0 is the best and 100 is the worst total score. The validity and reliability of this questionnaire has been reported previously. The test-retest reliability Pearson's correlation coefficients for the WOMAC items range from 0.55 to 0.78 , all being significant [39]. The measurement accuracy is to the one decimal point.

The Short-Form Health Survey (SF-36) was used to measure patient quality of life. It consists of eight domains (pain, physical function, general health perceptions, role limitation due to physical problems, role limitation due to emotional problems, emotional well-being, social functioning and energy/fatigue), which can be tallied to create a Physical Health Scale and a Mental Health Scale. Each measurement is scored from 0 to 100 , where 0 indicates the worst and 100 indicates the best patient health. The validity and reliability of this questionnaire has been reported previously. Reliability scores ranged from 0.76 to 0.93 . The scores for all scales met the customary level of scale reliability [32]. The measurement accuracy is to the one decimal point.

\section{Apparatus}

The Apos system and AposTherapy were used in the study (APOS-Medical and Sports Technologies Ltd. Herzliya, Israel). The device is comprised of two bulbous-shaped biomechanical elements attached to the sole of a shoe (Fig. 1). One biomechanical element is located under the hindfoot, and the other is located under the forefoot region. The elements are attached to the subject's foot via a platform in the form of a shoe. The platform is equipped with a specially designed sole, which consists of two mounting rails that enable flexible positioning of each element under each region. Each element can be individually calibrated to induce specific biomechanical challenges in multiple planes.

\section{Protocol}

All patients underwent measurement of height and weight. Patients then underwent a computerized gait test. During the test, all patients walked barefoot at a self-selected speed. The following parameters were evaluated: velocity $(\mathrm{cm} / \mathrm{s})$, step length $(\mathrm{cm})$ and SLS phase for each leg (\% gait cycle, GC). In addition, patients completed the WOMAC and SF-36.

After the first gait test, the biomechanical device was individually calibrated to each patient by a physiotherapist specialized in AposTherapy methodology. Therapy was then initiated and continued on a daily basis for a period of

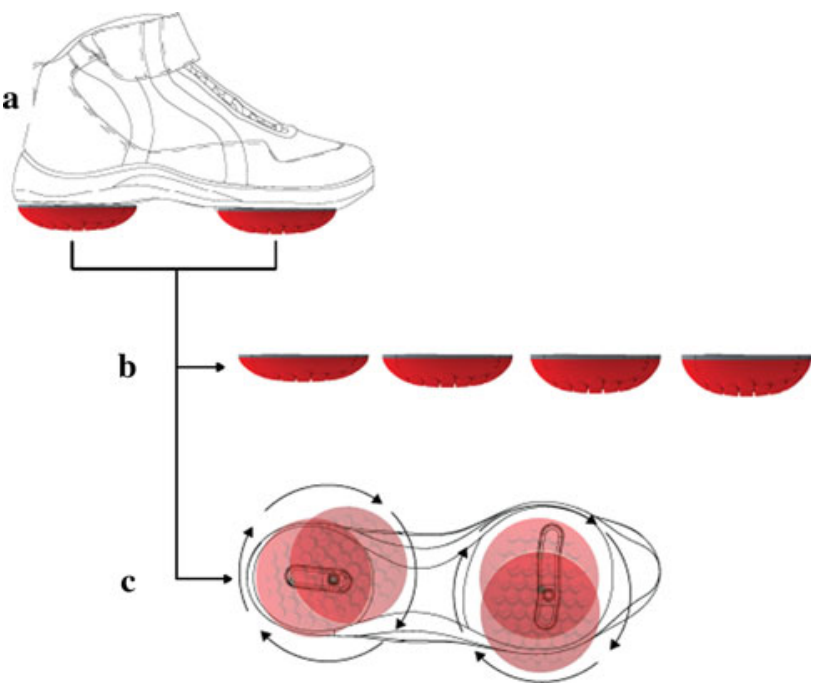

Fig. 1 Apos biomechanical system. a Biomechanical device comprising two individually calibrated elements and a foot-worn platform. The elements are attached to under the hindfoot and forefoot regions of the platform. b The biomechanical elements are available in different degrees of convexity and resilience. c The specially designed sole of the platform includes two mounting rails and a positioning matrix to enable flexible positioning of each biomechanical element

12 months. Patients were instructed to wear the device indoors as they perform their normal routines for an hour a day during the first week with an overall walking time of $10 \mathrm{~min}$ and to gradually increase walking time. All patients received a telephone call after the first and second weeks to verify compliance. A follow-up examination was conducted after 6 weeks in which patients were evaluated by the physiotherapist. After 3 and 12 months of therapy, patients underwent a gait test and completed the WOMAC and SF-36 questionnaires.

\section{Statistical analysis}

Data were analysed with SPSS software version 19.0. The significance levels were set at 0.05 . Data were presented as mean and standard deviation for continuous variables. Repeated-measures analyses were performed to compare gait parameters and self-evaluation questionnaires between baseline, and 3 and 12 months. Gait parameters and selfevaluation questionnaires following 1 year of therapy were presented by the mean and $95 \%$ confidence intervals. Furthermore, repeated-measures analysis with one nested variable (gender) was conducted to demonstrate the differences in improvement between genders.

A change in gait measurements was considered to be clinically significant as long as it was accompanied by an improvement in the WOMAC index that qualified under the Outcome Measures in Rheumatology Clinical Trials (OMERACT)-OARSI guidelines for clinical improvement [28] 


\section{Results}

All patients complied with the study protocol, and none reported any adverse events that disqualified them from the study. One patient chose to undergo knee arthroscopy and was considered as a failure to treatment. Significant differences were found in the patients' gait analysis after 12 months of therapy. Results are summarized in Table 1. In addition, significant improvement was noted in the levels of pain, function and stiffness, measured via the WOMAC index following 12 months of therapy (Table 2). The SF-36 Physical Health Scale and SF-36 Mental Health Scale (all $p<0.01$ ) both improved significantly following 12 months of therapy (Table 2).

A comparison between men and women was made. Women showed significantly lower gait velocity $(p=0.011)$ and shorter step length $(p=0.001)$ compared to men at baseline and following 12 months of therapy. SLS did not differ between men and women. Both genders significantly increased their gait velocity, step length and SLS following 12 months of therapy. Results are summarized in Table 3. In regard to quality of life, no gender differences were noted in the physical health score or in the mental health score. Both genders reported an improvement in quality of life following 12 months of therapy (Table 3 ). Figures 2 and 3 illustrate gender differences in WOMACpain and WOMAC-function throughout the study period, respectively.

A comparison between limbs was made for step length and SLS. No significant differences were found between involved step length and uninvolved step length at baseline and following therapy. Significant differences were seen

Table 1 Gait parameters changes following 12 months of therapy

\begin{tabular}{llccc}
\hline & Baseline & 3 months & 12 months & $p^{*}$ \\
\hline Velocity (cm/s) & $97.4(18.3)[90.9-103.9]$ & $112.0(18.3)[105.5-118.5]$ & $111.8(21.9)[104.0-119.5]$ & $<0.001$ \\
Involved step length (cm) & $55.2(7.7)[52.5-58.0]$ & $59.7(7.6)[57.0-62.3]$ & $59.1(10.0)[55.5-62.6]$ & 0.006 \\
Uninvolved step length (cm) & $55.8(8.4)[52.9-58.8]$ & $60.2(7.9)[57.4-63.0]$ & $60.3(9.6)[56.9-63.7]$ & 0.001 \\
Involved single-limb support (\% GC) & $37.6(2.3)[36.7-38.4]$ & $38.8(1.4)[38.3-39.3]$ & $38.9(1.7)[38.3-39.5]$ & 0.001 \\
Uninvolved single-limb support (\% GC) & $39.0(2.2)[38.2-39.7]$ & $39.2(1.7)[38.6-39.9]$ & $38.9(2.1)[38.2-39.6]$ & n.s. \\
\hline
\end{tabular}

Results are presented as mean (SD) [95\% confidence interval]

A significant improvement was seen after 3 months of therapy as well as after 12 months of therapy compared to the baseline examination, except for SLS of the uninvolved limb

$G C$ gait cycle

$* p$ value was set to $p<0.05$

Table 2 Changes in self-evaluation questionnaires following 12 months of therapy

\begin{tabular}{lllll}
\hline & Baseline & 3 months & 12 months \\
\hline WOMAC index (0-100 mm) & & & & \\
Pain & $42.8(21.5)[35.1-50.4]$ & $22.7(19.2)[15.9-29.5]$ & $11.7(14.0)[6.8-16.7]$ & $<0.001$ \\
Stiffness & $42.3(26.0)[33.1-51.5]$ & $20.5(18.5)[13.9-27.0]$ & $13.7(16.6)[7.8-19.6]$ & $<0.001$ \\
Function & $36.9(20.2)[29.7-44.0]$ & $20.5(17.4)[14.3-26.6]$ & $13.2(14.9)[7.9-18.5]$ & $<0.001$ \\
SF-36 health survey (0-100) & & & & \\
Physical function & $49.2(24.2)[40.6-57.8]$ & $61.5(21.4)[53.9-69.1]$ & $67.8(20.9)[60.5-75.3]$ & 0.001 \\
Pain & $43.0(20.8)[35.7-50.4]$ & $61.1(21.9)[53.3-68.8]$ & $65.9(23.8)[57.5-74.3]$ & $<0.001$ \\
Limitation due to physical health & $31.1(38.5)[17.4-44.7]$ & $58.3(41.3)[43.7-73.0]$ & $59.1(39.4)[45.1-73.1]$ & 0.001 \\
Energy/fatigue & $56.8(18.4)[50.3-63.3]$ & $64.8(15.1)[59.5-70.2]$ & $62.7(16.4)[56.9-68.6]$ & 0.04 \\
Emotional well-being & $71.3(15.6)[65.8-76.8]$ & $77.2(12.8)[72.7-81.8]$ & $75.9(12.6)[71.4-80.4]$ & n.s. \\
Limitation due to emotional problems & $55.6(46.2)[39.2-71.9]$ & $76.8(35.8)[64.1-89.5]$ & $70.7(38.0)[57.2-84.2]$ & n.s. \\
Social functioning & $68.2(20.5)[60.9-75.5]$ & $82.6(16.8)[76.6-88.5]$ & $86.0(13.9)[81.1-90.9]$ & $<0.001$ \\
General health & $58.3(17.9)[52.0-64.7]$ & $66.0(16.6)[60.1-71.9]$ & $65.3(12.4)[60.9-69.7]$ & 0.03 \\
Physical scale & $47.7(17.5)[41.5-53.9]$ & $62.4(17.9)[56.0-68.7]$ & $64.2(18.0)[57.8-70.6]$ & $<0.001$ \\
Mental scale & $62.0(17.0)[56.0-68.1]$ & $73.5(13.5)[68.7-78.3]$ & $72.1(13.5)[67.3-76.9]$ & 0.001 \\
\hline
\end{tabular}

Results are presented as mean (SD) [95\% confidence interval]

$* p$ value was set to $p<0.05$. A significant improvement was seen after 3 months of therapy as well as after 12 months of therapy compared to the baseline examination, except for emotional well-being and limitation due to emotional problems 


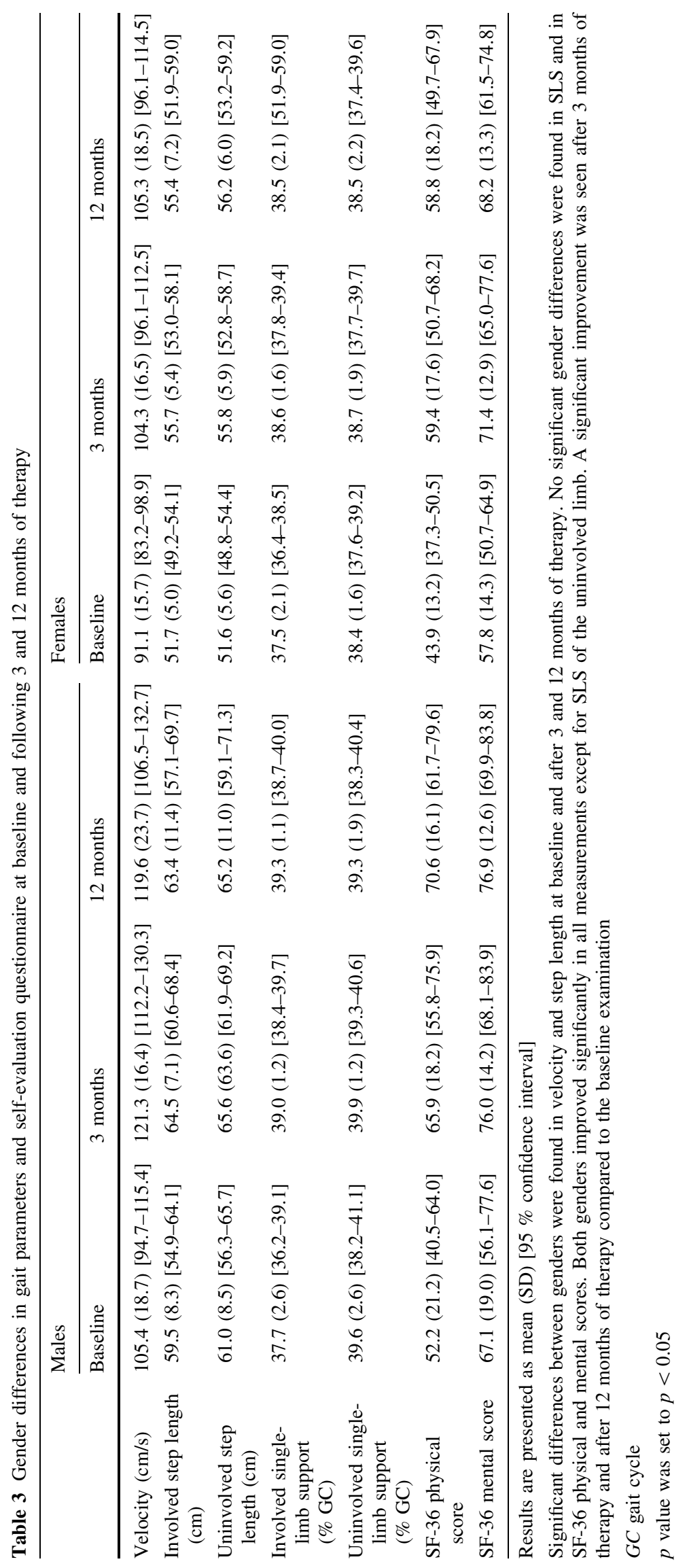




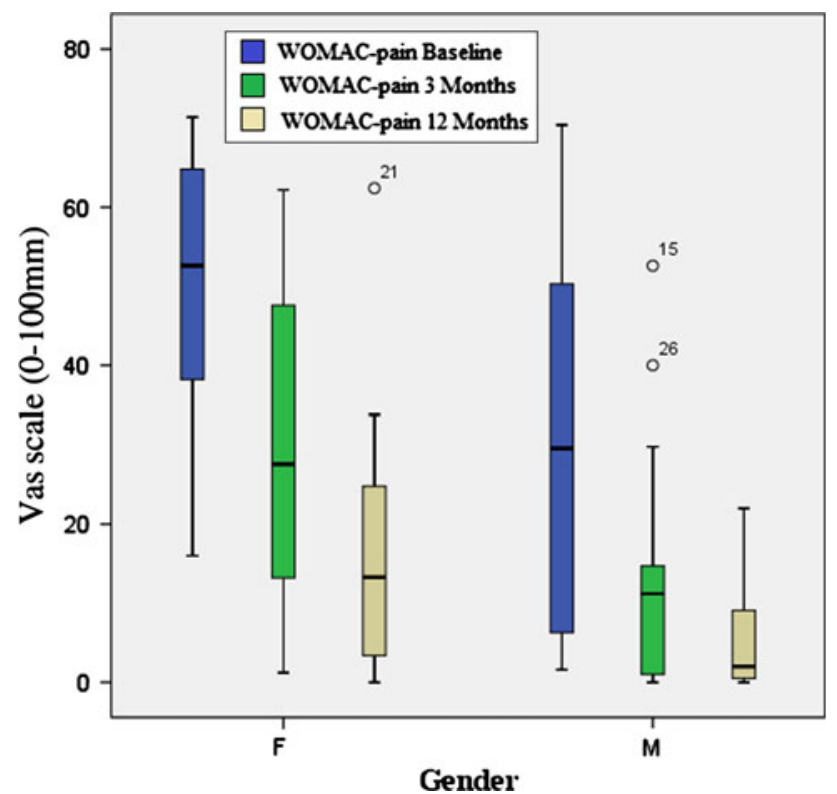

Fig. 2 WOMAC-pain changes following 12 months of therapy in women and men. Women had significantly higher levels of pain compared to men at all time points. Both women and men reported significant reduction in pain following 12 months of therapy

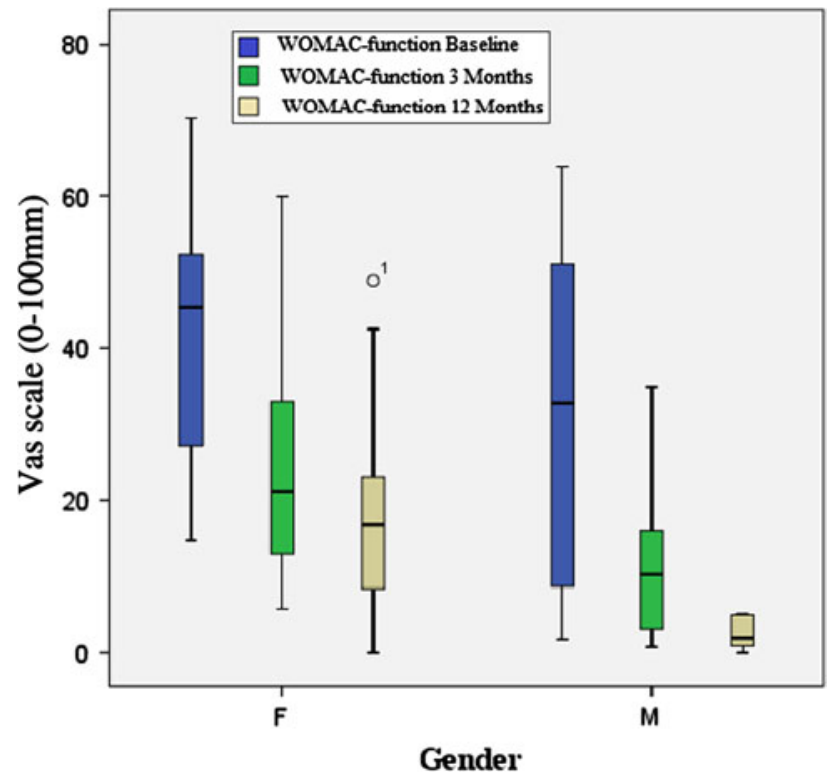

Fig. 3 WOMAC-function changes following 12 months of therapy in women and men. Women had significantly higher levels of functional limitation compared to men at all time points. Both women and men reported significant improvement in function following 12 months of therapy

between SLS of the involved limb compared to the SLS of the uninvolved limb at baseline $(p=0.013)$ and after 3 months of therapy $(p=0.023)$. There were no significant differences in SLS between limbs following 12 months of therapy.

\section{Discussion}

The most important finding of this study was the improvement in gait patterns of patients with degenerative meniscal tears after 3 months of therapy with a foot-worn biomechanical device. This improvement was accompanied by improvements in symptoms and quality of life. The present findings have relevance for the treatment for meniscal tears in conjunction with knee OA and suggest a potent new therapy for patients suffering from degenerative meniscal tears.

The improvements in gait patterns have significant implications in regard to the ability of this therapy to reestablish proper limb symmetry. A common shortcoming of both surgical and non-surgical therapies has been that proper limb symmetry during gait is rarely re-established. Asymmetry in the lower extremity exposes the body to unusual and potentially excessive loads, which has a negative impact on walking and potentially increases the risk of the development of knee OA [6, 7, 26, 31, 37]. The fact that SLS of the non-injured knee did not change is an important finding as it was already in normal range. Based on this finding, limb asymmetry was minimized by increasing the weight-bearing time of the injured knee without changing the non-injured knee.

The functional improvement in gait dynamics was also supported by the self-evaluation questionnaire data, which found significant improvements in physical function and pain as defined via the WOMAC index and the SF-36, meeting the OMERACT OARSI criteria for clinical response to a treatment [33]. These findings are unique as they match, if not exceed, similar self-evaluative questionnaire results following other therapies [8, 25, 27]. Though earlier works have found improvement in the WOMAC index and the SF-36 of patients following surgical and pharmaceutical therapy, AposTherapy demonstrated a positive quantitative degree of improvement in physical function and pain following non-invasive therapy [8, 24, 27]. Lastly, though our findings have important clinical significance for the relatively short-term rehabilitation of meniscal tears, attention must be paid to the long-term implications. Numerous studies have related meniscus injuries with the development of knee OA [6, 26, 28]. The implemented therapy of this study has been shown to have a positive therapeutic effect on the gait pattern, physical function and pain of patients with knee OA $[3,11,17,20]$. Therefore, it can be suggested that this treatment modality has the potential to be beneficial in two timeframes: the short-term rehabilitation of patients with meniscal tears and the longterm treatment for knee OA. These findings suggest that the use of this foot-worn biomechanical device may serve as an additional conservative treatment modality for patients with a degenerative meniscal tear and might reduce the need for surgery in these patients. Furthermore, since treatment is 
performed in the patient's own environment while performing daily activities, high compliance is expected and the patient is driven to take responsibility for his recovery.

This study had some limitations. First, the present study lacked a control group; secondly, therapy did not commence immediately following the injury but within a 3 -month time window. It is known, however, that in most cases, if a patient does not feel alleviation in pain within 3 months of injury, he or she will be recommended for surgery. It can be assumed that the patients in the present study received the standard care of treatment before commencing the biomechanical therapy and were recommended to undergo arthroscopy. Further studies should examine the effect of this therapy immediately after injury to see whether it can accelerate the rehabilitation period, as well as compare the therapy-compatible control group. Researchers should also consider comparing the outcomes of this conservative treatment with the outcomes of surgical procedures for meniscal injuries.

\section{Conclusions}

Knee OA patients with degenerative meniscal tears treated with AposTherapy for 12 months demonstrated improved gait patterns (increased walking velocity, longer step length and higher SLS). Furthermore, patients also showed improved limb symmetry following the therapy. Finally, a statistically and clinically significant improvement was found in physical function and pain as measured by two different self-evaluation questionnaires, the WOMAC index and the SF-36.

Acknowledgments The authors thank Nira Koren-Morag, $\mathrm{PhD}$, for statistical analysis assistance and Lior Atlas for data collection assistance.

Conflict of interest Avi Elbaz, Amit Mor and Ronen Debi hold shares in AposTherapy. Ganit Segal is a salaried employee of AposTherapy. Yiftah Beer, Ehud Rath, Guy Morag, Daniel Wasser and Eytan Debbi are co-researchers in a number of studies. They do not receive and are not entitled to any financial compensation from AposTherapy.

Open Access This article is distributed under the terms of the Creative Commons Attribution License which permits any use, distribution, and reproduction in any medium, provided the original author(s) and the source are credited.

\section{References}

1. Altman R, Asch E, Bloch D et al (1986) Development of criteria for the classification and reporting of osteoarthritis. Classification of osteoarthritis of the knee. Diagnostic and therapeutic criteria
Committee of the American Rheumatism Association. Arthr Rheum 29(8):1039-1049

2. Andersson-Molina H, Karlsson H, Rochborn P (2002) Arthroscopic partial and total meniscectomy: a long-term follow-up study with matched controls. Arthroscopy 18(2):183-189

3. Bar-Ziv Y, Beer Y, Ran T et al (2010) A treatment applying a biomechanical device to the feet of patients with knee osteoarthritis results in reduced pain and improved function: a prospective controlled study. BMC Musculoskelet Disord 11:179

4. Barker S, Craik R, Freedman W et al (2006) Accuracy, reliability, and validity of a spatiotemporal gait analysis system. Med Eng Phys 28(5):460-467

5. Behairy NH, Dorgham MA, Khaled SA (2009) Accuracy of routine magnetic resonance imaging in meniscal and ligamentous injuries of the knee: comparison with arthroscopy. Int Orthop 33(4):961-967

6. Bhattacharyya T, Gale D, Dewire P et al (2003) The clinical importance of meniscal tears demonstrated by magnetic resonance imaging in osteoarthritis of the knee. J Bone Joint Surg Am 85-A(1):4-9

7. Bulgheroni P, Bulgheroni MV, Ronga M et al (2007) Gait analysis of pre- and post-meniscectomy knee: a prospective study. Knee 14(6):472-477

8. Busija L, Osborne RH, Nilsdotter A et al (2008) Magnitude and meaningfulness of change in SF-36 scores in four types of orthopedic surgery. Health Qual Life Outcomes 6:55

9. Debi R, Mor A, Segal G et al (2011) Correlation between single limb support phase and self-evaluation questionnaires in knee osteoarthritis populations. Disabil Rehabil 33:1103-1109

10. Elbaz A, Mirovsky Y, Mor A et al (2009) A novel biomechanical device improves gait pattern in patient with chronic nonspecific low back pain. Spine (Phila Pa 1976) 34(15):E507-E512

11. Elbaz A, Mor A, Segal G et al (2010) APOS therapy improves clinical measurements and gait in patients with knee osteoarthritis. Clin Biomech (Bristol, Avon) 25(9):920-925

12. Elbaz A, Mor A, Segal O et al (2011) Can single limb support objectively assess the functional severity of knee osteoarthritis? Knee 19:32-35

13. Englund M, Guermazi A, Gale D et al (2008) Incidental meniscal findings on knee MRI in middle-aged and elderly persons. N Engl J Med 359(11):1108-1115

14. Englund M, Guermazi A, Roemer FW et al (2009) Meniscal tear in knees without surgery and the development of radiographic osteoarthritis among middle-aged and elderly persons: the multicenter osteoarthritis study. Arthr Rheum 60(3):831-839

15. Ericsson YB, Dahlberg LE, Roos EM (2009) Effects of functional exercise training on performance and muscle strength after menisectomy: a randomized trial. Scand J Med Sci Sports 19: $156-165$

16. Garrett WE, Swiontkowski MF, Weinstein JN et al (2006) American board of orthopaedic surgery practice of the orthopaedic surgeon: part-II, certification examination case mix. J Bone Joint Surg Am 88(3):660-667

17. Goryachev Y, Debbi EM, Haim A et al (2011) Foot center of pressure manipulation and gait therapy influence lower limb muscle activation in patients with osteoarthritis of the knee. J Electromyogr Kinesiol 21(5):704-711

18. Haim A, Rozen N, Dekel S et al (2008) Control of knee coronal plane moment via modulation of center of pressure: a prospective gait analysis study. J Biomech 41(14):3010-3016

19. Haim A, Rozen N, Wolf A (2010) The influence of sagittal center of pressure offset on gait kinematics and kinetics. J Biomech 43(5):969-977

20. Haim A, Rubin G, Rozen N et al (2011) Reduction in knee adduction moment via non-invasive biomechanical training: a longitudinal gait analysis study. J Biomech 45:41-45 
21. Haim A, Wolf A, Rubin G et al (2011) Effect of center of pressure modulation on knee adduction moment in medial compartment knee osteoarthritis. J Orthop Res 29(11):1668-1674

22. Han SB, Shetly GM, Lee DH et al (2010) Unfavorable results of partial menisectomy for complete posterior medial meniscus root tear with early osteoarthritis: a 5- to 8-year follow-up study. Arthroscopy 26(10):1326-1332

23. Harding VR, Williams AC, Richardson PH et al (1994) The development of a battery of measures for assessing physical functioning of chronic pain patients. Pain 58(3):367-375

24. Herrlin S, Hallander M, Wange P et al (2007) Arthroscopic or conservative treatment of degenerative medial meniscal tears: a prospective randomised trial. Knee Surg Sports Traumatol Arthrosc 15(4):393-401

25. Hong CL, Bae JH, Wang JO et al (2010) Non-operative treatment of degenerative posterior root tear of the medial meniscus. Knee Surg Sports Traumatol Arthrosc 18(4):535-539

26. Hunter DJ, Zhang YQ, Niu JB et al (2006) The association of meniscal pathologic changes with cartilage loss in symptomatic knee osteoarthritis. Arthr Rheum 54(3):795-801

27. Huskin JP, Vandekerckhove B, Delince P et al (2008) Multicentre, prospective, open study to evaluate the safety and efficacy of hylan G-F 20 in knee osteoarthritis subjects presenting with pain following arthroscopic meniscectomy. Knee Surg Sports Traumatol Arthrosc 16(8):747-752

28. Jee WH, McCauley TR, Kim JM et al (2003) Meniscal tear configurations: categorization with MR imaging. AJR Am J Roentgenol 180(1):93-97

29. Khodadadeh S, Eisenstein SM (1993) Gait analysis of patients with low back pain before and after surgery. Spine (Phila Pa 1976) 18(11):1451-1455

30. Kim JH, Chung JH, Lee DH et al (2011) Arthroscopic suture anchor repair versus pullout suture repair in posterior root tear of the medial meniscus: a prospective comparison study. Arthroscopy 27(12):1644-1653

31. Lee DH, Lee BS, Kim JM et al (2011) Predictors of degenerative medial meniscus extrusion: radial component and knee osteoarthritis. Knee Surg Sports Traumatol Arthrosc 19(2):222-229

32. Lewin-Epstein N, Sagiv-Schifter T, Shabtai EL et al (1998) Validation of the 36-item short-form health survey (Hebrew version) in the adult population of Israel. Med Care 36:13611370

33. Pham T, Van der Heijde D, Altman RD et al (2004) OMERACTOARSI initiative: osteoarthritis research society international set of responder criteria for osteoarthritis clinical trials revisited. Osteoarthr Cartil 12(5):389-399

34. Rodkey WG (2000) Basic biology of the meniscus and response to injury. Instr Course Lect 49:189-193

35. Roos H, Lauren M, Adalberth T et al (1998) Knee osteoarthritis after meniscectomy: prevalence of radiographic changes after twenty-one years, compared with matched controls. Arthr Rheum 41(4):687-693

36. Saxon L, Finch C, Bass S (1999) Sports participation, sports injuries and osteoarthritis: implications for prevention. Sports Med 28(2):123-135

37. Sturnieks DL, Besier TF, Mills PM et al (2008) Knee joint biomechanics following arthroscopic partial meniscectomy. J Orthop Res 26(8): 1075-1080

38. Viljakka T (1997) The clinical diagnosis and results of conservative treatment of knee distortion injury. Arch Orthop Trauma Surg 116(1-2):6-9

39. Wigler I, Neumann L, Yaron M (1999) Validation study of a Hebrew version of WOMAC in patients with osteoarthritis of the knee. Clin Rheumatol 18:402-405 